\title{
ANÁLISIS DE LA EXPERIENCIA SUBJETIVA EN MUJERES CON CÁNCER DE MAMA
}

\section{SUBJECTIVE EXPERIENCE ANALYSIS IN WOMEN WITH BREAST CANCER}

\author{
TÍTULO CORTO: ANÁLISIS DE LA EXPERIENCIA SUBJETIVA EN MUJERES
}

Miriam Belber-Gómez $^{1 \mathbb{D}}$, Fátima Valencia-Agudo ${ }^{(\mathbb{D}}$, María Eugenia De la Viuda-Suárez $^{3}$ (D)

Tipología: Artículo de Investigación Científica y Tecnológica

Para citar este artículo: Belber-Gómez M, Valencia-Agudo F, De la Viuda-Suárez ME. Análisis de la experiencia subjetiva en mujeres con cáncer de mama. Duazary. 2018 enero; 15 (1): 71 - 85.

Doi: http://dx.doi.org/10.21676/2389783X.2021

Recibido en agosto 12 de 2016

Aceptado en marzo 02 de 2017

Publicado en línea en septiembre 13 de 2017

\section{RESUMEN}

En este artículo se analiza la experiencia y necesidades psicológicas presentes en el discurso de las participantes durante una intervención psicoterapéutica grupal dirigida a mujeres con cáncer de mama. Se transcriben las sesiones del grupo y se realiza un análisis del discurso, recogiendo los temas más prevalentes. Las principales dificultades psicológicas recogidas son el cambio en su imagen corporal y su sexualidad, la nueva vivencia de las relaciones interpersonales, las repercusiones del discurso social sobre la importancia de las emociones positivas, el miedo a la recidiva, las relaciones con el personal sanitario y los cambios tras la enfermedad. Se recogen aspectos que las pacientes consideran factores de ayuda en el grupo (sentirse comprendidas, ver a las compañeras como modelos de afrontamiento o cambiar su relación con la enfermedad). Se apuntan implicaciones clínicas para una mejora en la atención integral a las pacientes con cáncer de mama.

Palabras clave: Psicoterapia de grupo; Neoplasias de la mama; Salud mental; Atención plena; Investigación cualitativa.

\begin{abstract}
In this article the psychological experience and needs shown in the discourse of women diagnosed with breast cancer in a psychological group intervention were analyzed. The sessions are transcribed and a discourse analysis is performed, selecting the most prevailing topics. The main psychological difficulties perceived by the participants

1. Licenciada en Psicología, especialista en Psicología Clínica. Hospital Universitario Son Espases. España. Correo: miriambelber@gmail.com - http:// orcid.org/0000-0002-7691-7371

2. Licenciada en Psicología, especialista en Psicología Clínica. Hospital Universitario Central de Asturias. España. Correo: fatima-valencia@hotmail.es http://orcid.org/0000-0001-6097-6716

3. Licenciada en Psicología, especialista en Psicología Clínica. Hospital Universitario Central de Asturias. España. Correo: euvisu@hotmail.com - http:// orcid.org/0000-0003-0642-2290
\end{abstract}


are the following: body identity change, sexuality changes, new quality of interpersonal relationships, implications of positive thinking culture, fear of recurrence, the relationship with the hospital staff, and change after diagnosis. The aspects that the group considered helpful are also addressed, i.e. feeling understood by the others, seeing the rest of participants as coping models, changing their relationship with the illness. Several clinical implications are highlighted in order to improve a comprehensive care.

Keywords: Psychotherapy Group; Breast Neoplasms; Mental Health; Mindfulness; Qualitative Research.

\section{INTRODUCCIÓN}

$E^{1}$ cáncer de mama es el tipo de cáncer más frecuente entre las mujeres de todo el mundo y la principal causa de muerte por cáncer en las mujeres europeas. Se estima que el riesgo de padecer cáncer de mama a lo largo de la vida es de una de cada ocho mujeres ${ }^{1}$.

Gracias a los avances médicos en el tratamiento y detección temprana de este tipo de enfermedades, la supervivencia ha aumentado de una manera relevante. Si bien a nivel fisiológico parece que cada vez hay mayores avances, a nivel psicológico el cáncer supone grandes costes personales y sociales, somete a las personas afectadas y su familia a un gran número de sucesos estresantes asociados al diagnóstico y tratamiento, durante intervalos largos de tiempo, que amenazan su trayectoria vital ${ }^{2}$.

Los procesos psicológicos por los que pasa una persona al ser diagnosticada con cáncer tienen una serie de particularidades. La experiencia de enfermedad y cercanía a la muerte hace que los esquemas que componen la propia identidad del "yo" se vean amenazados y tengan que volver a reconstruirse. La lucha contra la enfermedad altera la percepción del presente y del futuro ${ }^{3}$.

Por ello, el objetivo psicoterapéutico principal debe dirigirse a facilitar la adaptación a una situación cambiante y de alto riesgo, fortaleciendo la percepción de controlabilidad y otros recursos, tanto internos como externos, de tal modo que la percepción subjetiva de sufrimiento disminuya y aumente la capacidad de afrontamiento adaptativo. Se hace necesaria una intervención dirigida a reafirmar y normalizar sus sentimientos, reconocer y validar las necesidades de expresión emocional y de integración de los "pedacitos" del yo que han quedado quebrados tras la experiencia de cáncer ${ }^{3}$.

Se han propuesto diferentes formas de apoyo psicológico y terapias ${ }^{4-7}$ que pueden mejorar la calidad de vida de los pacientes oncológicos y facilitar el proceso de adaptación a la enfermedad. Dentro de las propuestas de apoyo y reintegración psicológica se encuentra la psicoterapia grupal. La literatura muestra una mejoría de los grupos de terapia en pacientes con cáncer, incluyendo mejora en el estado de ánimo, capacidad de afrontamiento y ajuste al cáncer y al dolor. Este tipo de intervención facilita el cambio de actitudes, conductas y emociones, a través de la reflexión conjunta, el intercambio de experiencias, el autoconocimiento y el apoyo mutuo ${ }^{8}$.

En cuanto al tipo de metodología que emplea este tipo de estudios, suele utilizarse frecuentemente la cuantitativa. Sin embargo, en el ámbito del cáncer existen trabajos que se encargan de recoger de forma directa las vivencias personales en torno a la enfermedad y las necesidades psicológicas de las pacientes ${ }^{9-12}$. En síntesis, serían cuatro áreas las que se ven mayormente afectadas en supervivientes de cáncer de mama: 
la conciencia sobre la propia muerte, la vivencia de una certera incertidumbre, la validación de los vínculos de apego y la redefinición de la identidad del yo ${ }^{13}$.

Si se tiene en consideración lo expuesto, se hace necesario un abordaje psicoterapéutico integral donde primen y se tengan en cuenta las necesidades psicológicas de las personas. Para ello, se entiende como fundamental atender a la experiencia de las propias pacientes, conociendo sus percepciones en torno a las cuestiones que les generan mayor malestar emocional y acerca de las intervenciones que valoran de forma positiva.

Mediante el presente trabajo se pretenden los siguientes objetivos: analizar de manera cualitativa la experiencia de las mujeres con cáncer de mama en torno a la enfermedad, los tratamientos y el malestar psicológico; recoger las necesidades psicológicas y clínicas que reflejan en su discurso; y plantear y reflexionar sobre posibles propuestas terapéuticas en base al análisis de su discurso y necesidades.

\section{MATERIALES Y MÉTODOS}

\section{Muestra}

Las participantes fueron todas mujeres que padecían un proceso de cáncer de mama. Los criterios de inclusión fueron padecer un proceso oncológico diagnosticado en los últimos 18 meses, estar recibiendo atención psicológica en el Servicio de salud mental de enlace del Hospital Universitario Central de Asturias (HUCA) e interés en participar. Los criterios de exclusión fueron diagnósticos recientes (menos de un mes) y padecer un trastorno mental grave.

Asistieron al tratamiento grupal un total de seismujeres de las cuales cinco finalizaron el programa psicoterapéutico.
El rango de edad de las participantes estaba comprendido entre los 35 y los 64 años ( $X=46,5$ años, DT $=10,76)$. El $66,6 \%$ de las mujeres estaban casadas mientras que el 33,3\% estaban en situación de soltería. Al inicio del grupo casi las tres cuartas partes de las usuarias estaban en situación de baja laboral por enfermedad $(66,6 \%)$ mientras que una de ellas había perdido su trabajo y otra ya se había incorporado a su puesto habitual.

Respecto a los tratamientos aplicados, el 83,3 $\%$ seguía recibiendo tratamiento activo en el momento de comenzar el grupo mientras que el $16,6 \%$ ya no estaba recibiendo ningún tipo de tratamiento.

\section{Procedimiento}

Las evaluaciones y las sesiones grupales fueron realizadas en las instalaciones del Servicio de salud mental de enlace del HUCA. El programa grupal fue aplicado por tres Psicólogas Internas Residentes (PIR) bajo la supervisión de Psicólogos Clínicos pertenecientes al servicio.

La elección de las participantes se realizó por parte de los profesionales del Servicio de salud mental de enlace del HUCA. Seleccionaron a aquellas pacientes que estaban recibiendo tratamiento psicológico que se adecuaban a los criterios de exclusión e inclusión.

Antes de consolidar el grupo se les aplicó una pequeña entrevista semiestructurada donde se recogían aspectos referentes a variables sociodemográficas y clínicas. Se les explicó de manera breve el funcionamiento del grupo y se aprovechó para resolver las dudas que pudieran surgir. Además, se les solicitó consentimiento informado por escrito.

Se aplicó un programa de tratamiento en modalidad grupal en la sala de grupos del 
servicio. Esta sala disponía de un espejo unidireccional para investigar los tratamientos grupales. Se aplicaron 10 sesiones cuya duración era de 90 minutos y con una frecuencia semanal. El formato del grupo fue cerrado al iniciar el primer módulo, aunque al comenzar el segundo se pudieron incorporar más participantes. La disposición de las participantes en el espacio era en círculo para favorecer la comunicación entre las usuarias. El formato fue semiestructurado introduciendo breves temas teóricos sobre un aspecto a trabajar, ejercicios de reflexión, prácticas experienciales y resolución de dudas.

Una vez finalizado el programa se les pedía a las usuarias que cubrieran de manera anónima una pequeña encuesta de satisfacción con el tratamiento grupal.

\section{Evaluación}

En la última fase se aplicó una encuesta para medir el nivel de satisfacción con el tratamiento. Es una encuesta anónima con tres ítems referentes a la valoración de los aspectos más positivos y negativos del grupo y un espacio para sugerencias y opiniones personales.

A su vez, cada sesión era transcrita mediante la observación detrás del espejo anotando las dinámicas que surgían en el grupo, los temas, preocupaciones y sugerencias que realizaban las usuarias. Todo ello quedaba registrado para su posterior análisis.

\section{Intervención}

El programa terapéutico fue creado basándose en materiales relacionados con tratamientos psicológicos grupales en pacientes con cáncer de mama y Terapias de Tercera Generación (TTG) aplicadas a este tipo de población. Se decidió dividir el programa en dos módulos: 1) el primero consistía en trabajar aquellos aspectos psicológicos que más les preocupan a las pacientes con cáncer de mama utilizando una orientación basada en filosofía de la Terapia de Aceptación y Compromiso (ACT); 2) en el segundo bloque se introducía la práctica de Mindfulness.

La estructura utilizada en las sesiones siempre era la misma: primero se resolvían dudas y se reflexionaba brevemente sobre la sesión previa; después se hacía una introducción teórica sobre un tema nuevo a trabajar; se hacían ejercicios prácticos; y finalmente se cerraba la sesión con un espacio de reflexión y con las conclusiones principales sobre el taller.

La orientación escogida era acorde a la filosofía de las TTG, es decir, se utilizaban las premisas de no juzgar, dejar fluir, aceptación incondicional de las usuarias y sus comentarios además de favorecer la ventilación emocional $\mathrm{y}$ validar todas las expresiones que realizaban las usuarias.

\section{Declaración sobre aspectos éticos}

Se tuvo en cuenta lo dispuesto en la Declaración de Helsinki. Se les presentó el proyecto grupal y, una vez explicado y comprendido, se diligenció el formato de consentimiento informado por escrito, en el cual daban su autorización para que las sesiones fuesen supervisadas detrás del espejo unidireccional. Todas las participantes autorizaron la supervisión, trascripción y análisis de las sesiones para su posterior utilización con fines docentes o de investigación. Se garantizó la protección a la intimidad, a la confidencialidad y el derecho al anonimato tanto de los datos como de las entrevistas. Por tanto, este estudio respeta los principios éticos de las participantes. 


\section{RESULTADOS}

Estudio descriptivo de metodología cualitativa en el que se analiza el discurso de las participantes por medio de las transcripciones, así como la impresión subjetiva de cada de ellas en la encuesta de satisfacción.

\section{Análisis del discurso}

Se exponen las cuestiones principales que aparecen en el discurso sostenido por las pacientes.

\section{Dificultades de afrontamiento de la imagen corporal y problemas en la sexualidad}

Todas las participantes coinciden en señalar lo complicado que les está resultando enfrentarse a su nueva imagen corporal. Una de las mayores dificultades está en relación a la cirugía, especialmente cuando ésta es radical. Algunas de ellas refieren no mirarse al espejo, llegando a colocar una toalla para taparlo cuando van a ducharse, y no haberse mostrado desnuda ante su pareja. Las pacientes dicen verse "como un cuadro abstracto" y en repetidas ocasiones se refieren a la operación "como una mutilación". Otros cambios físicos que tuvieron un gran impacto en ellas fueron la caída del pelo y el aumento de peso.

Además de las consecuencias emocionales, todo esto tiene efectos en sus relaciones sociales, dado que evitan determinadas situaciones para que los demás no vean su aspecto. Con frecuencia temen que otras personas se den cuenta de la mastectomía y refieren episodios en los que están con alguien, pero se encuentran continuamente auto-observándose y preguntándose qué estarán pensando los otros.

Los cambios físicos tienen además efectos en su identidad como persona, y sobre todo como mujer. Como se verá más adelante, son frecuentes las referencias a "ya no ser la misma".

Sin embargo, a lo largo del desarrollo de las sesiones, las pacientes van contando sus avances en torno a este tema. Cómo se han atrevido a mirarse y no ha sido tan terrible o cómo han podido construir significados en torno a la situación que les ayuden a salir adelante. Por ejemplo, una de ellas, que había sufrido mucho en relación al cuerpo y hablaba de "mutilación", cuenta que ha empezado a plantearse que pese al cambio corporal sigue siendo ella y que las intervenciones quirúrgicas fueron necesarias para salvar su vida.

En cuanto a la sexualidad, comentan que es un tema del que no habían podido hablar con nadie, y para muchas de ellas es en el espacio del grupo cuando pueden hacerlo por primera vez. Se trata de algo tabú, algo que nadie plantea, que no sale en televisión cuando se aborda el cáncer de mama. Es un tema del que tienen muchas dudas y refieren no haber sido informadas de los efectos que los tratamientos podrían tener en el deseo sexual o en la respuesta de excitación. De alguna forma, se les ha insistido desde el sistema sanitario que no deben preocuparse por eso (al igual que ocurre con la imagen corporal), ya quelo fundamental es sobrevivir. Parece que ni siquiera es un tema que traten con sus parejas. Además de dificultades relacionadas con los efectos físicos de los tratamientos, también reconocen la influencia de afectaciones psicológicas, como por ejemplo el miedo al rechazo por parte de sus parejas o el no verse atractivas.

\section{Vivencias en torno a las relaciones interpersonales}

Las pacientes comentan con frecuencia experiencias tanto positivas como negativas en la relación con otras personas tras la aparición de la enfermedad. Por una parte, refieren situaciones 
en las que sintieron incomprensión y banalización de lo que sentían por parte de los demás. Ellas mismas reconocen en muchos casos que es probable que la otra persona no hiciese o dijese algo con mala intención, pero ponen de manifiesto las dificultades del entorno para adaptarse a sus necesidades. Una paciente lamenta que cada vez que habla del tema le dicen que no se preocupe y que se anime, cuando para ella el estado de ánimo no es algo que dependa completamente de su voluntad. En otros casos, personas cercanas insisten en hablar del tema y preguntan cómo se encuentran, cuando ellas prefieren hablar de otras cosas o distraerse durante un rato de su situación.

Algunas de las mujeres refieren comentarios desagradables, inapropiados y preguntas indiscretas acerca de su estado físico. Asimismo, parece que varias de ellas sufrieron experiencias en las que otras personas las culpabilizaron o responsabilizaron de sufrir la enfermedad por el estilo de vida previo que tenían.

A pesar de su percepción de que la familia no siempre sabía cómo apoyarlas, las participantes sí valoraban tenerles ahí y reconocían la parte en la que sí habían sido ayudadas de forma muy positiva. Esto se puso especialmente de manifiesto ante una de las tareas que se sugirieron en el grupo, que consistió en pedir a alguien significativo que escribiese en un sobre cerrado una carta contando qué cosas valoraba y admiraba de la paciente, momentos importantes que habían vivido juntos, etc. Las cartas fueron leídas en la sesión y el resultado fue una experiencia muy emocionante, en la que fue patente que todas ellas, a pesar de las dificultades, tenían alguna figura de apego estable en la que apoyarse en momentos difíciles.

\section{Repercusión del discurso social acerca de las emociones positivas}

Las pacientes del grupo comentan en repetidas ocasiones el malestar que les produce que las animen a encontrarse contentas e incluso a ver la enfermedad como una oportunidad, dado que, de esta forma, favorecerán la recuperación. Todas coinciden en señalar que el cáncer no puede ser visto como algo positivo. Algunas de ellas han llegado a experimentar situaciones en las que se les responsabilizaba de su enfermedad, como es el caso de una paciente que cuenta que su madre le dijo que "esto se lo había causado ella misma por su pesimismo”.

Son frecuentes también los sentimientos de rabia e indignación, planteando que no es posible ver en el cáncer algo bueno. Hacen referencia a la imagen distorsionada que se ofrece por parte de algunos personajes famosos que han sufrido esta enfermedad, ya que se presentan como triunfadores, con una vida enriquecida $y$ mejorada tras el proceso, y sin marcas visibles del cáncer ni de sus tratamientos.

\section{Relación con el personal sanitario y necesidades en dicho contexto}

En cuanto a la relación con los profesionales, parecen haber vivido situaciones muy dispares. Por un lado, hubo momentos en los que se sintieron bien atendidas y personas con las que incluso llegaron a establecer un vínculo emocional y que fueron un apoyo en momentos difíciles. Tal es el caso de una enfermera que ayudó a una paciente a mirarse al espejo tras la mastectomía, y con la que pudo llorar y expresarse. Por otra parte, fueron frecuentes los relatos de experiencias de intenso malestar en el contexto del hospital. En general, las pacientes perciben falta de información acerca de aspectos prácticos y de cuidado personal cuando están llevando a cabo un determinado tratamiento (sujetadores, pelucas, ejercicio, etc.). En el grupo ponen en común diferentes pautas que les han dado acerca de alguna determinada cuestión, algunas veces contradictorias. Sienten además desinterés del personal por dar solución a sus 
preguntas y dudas sobre estos temas (lo cual quizás está motivado por sus dificultades para abordarlos con las pacientes).

Otra preocupación importante es la sensación percibida de pérdida de intimidad y de despersonalización en el hospital, de ser un número de historia clínica más. Comentan experiencias en las que el personal les pidió desnudarse en áreas que no aseguraban lo suficiente la privacidad, lo cual fue vivido como una falta de respeto.

\section{Cambio en la identidad}

La mayoría eran mujeres muy activas antes del diagnóstico, con un importante nivel de responsabilidad. Trabajaban, cuidaban de los demás y disfrutaban del ocio. En estos momentos tienen dificultades para "reconocerse a sí mismas", ya que en muchos casos han perdido el rol con el que más se identificaban. En el ámbito laboral, la mayoría están de baja y una de ellas perdió su trabajo tras el diagnóstico. Se plantean qué sucederá en el futuro y cómo podrían replantearse su vida, dado que a algunas las secuelas les impedirán retomar la actividad previa.

Los estados emocionales que sienten en la actualidad fomentan esta sensación de cambio. Si antes estaban alegres, satisfechas con sus vidas y con planes y proyectos, ahora se encuentran tristes, apáticas y, en ocasiones, con una visión restringida del futuro. Esto hace que digan cosas como "ya no soy la misma" o "no me reconozco". Son frecuentes las referencias al fracaso.

En las últimas sesiones se perciben cambios en el discurso del grupo en relación a este tema. La paciente que había perdido el trabajo cambia su actitud y plantea la nueva situación como algo a lo que ha de hacer frente de la mejor manera posible, "hay que reinventarse". Se genera un discurso en el que se reconoce que tras esta experiencia "es imposible volver a ser la misma", pero "nadie puede decir que en ese cambio tenga que ser todo negativo".

\section{Cambios tras la enfermedad}

Varias de las pacientes se muestran reticentes a reconocer cambios positivos en su entorno y en sus capacidades de afrontamiento tras la aparición de la enfermedad. Una cuestión en la que coincidía la mayoría fue en afrontar de forma activa situaciones sociales en las que antes se mostraban inhibidas. Refieren mayor capacidad para responder ante los abusos, ya sea en el ámbito laboral o en el entorno de la familia.

Plantean también el no dar tanta importancia a cosas que no la tienen, preocupándose solo por temas relevantes y reconociendo que determinadas personas de su entorno presentan limitaciones que tendrán que aceptar. En relación con esto, se observa que utilizan en mayor medida el humor al enfrentarse a los problemas.

En cuanto a las relaciones sociales, parece que esta experiencia les ha ayudado a discriminar entre aquellas personas en las que pueden confiar y aquellas con las que es necesario guardar cierta cautela. En ciertos casos, la aparición de la enfermedad dio lugar a una mayor intimidad en las relaciones interpersonales. Este es el caso de una paciente y su pareja, que decidieron irse a vivir juntos después de años de relación.

Otro aspecto observado fue una mayor apreciación del presente y por las experiencias del ahora. Esto se puso de manifiesto por ejemplo cuando una paciente cuenta que se despierta por la mañana y piensa "qué bien que sigo aquí", o cuando refieren fijarse en los árboles o el paisaje al ir dando un paseo, o cuando una de ellas comenta que salió a la calle sin paraguas porque le apetecía sentir la lluvia. 


\section{Encuesta de satisfacción}

En la encuesta las pacientes señalan entre los aspectos más positivos: conocer personas que atraviesan por una situación parecida, intercambiar experiencias, disminuir la ansiedad y el miedo, reducir la sensación de soledad, ver que la vida continúa, aumentar la confianza en una misma y poder ayudar a otras mujeres. En relación a los aspectos negativos plantean: el miedo al grupo antes de conocer al resto de participantes y ver que cada caso es diferente, aunque se trate de la misma enfermedad.

En lo que se refiere a las sugerencias, las pacientes plantearon: mayor número de sesiones, mejorar la coordinación entre diferentes departamentos del hospital, información acerca de temas que les generan dudas, realizar un tríptico informativo con aspectos físicos y psicológicos relacionados con la enfermedad y el tratamiento y generar grupos informales de apoyo mutuo.

\section{DISCUSIÓN}

Del relato de las pacientes se pueden concluir las necesidades que se enuncian a continuación.

En relación a la imagen corporal y sexualidad se encontró que se trata de una temática que les preocupa y a la que muchas veces no se le da la importancia suficiente desde los servicios sanitarios. La investigación indica los efectos que provocan en la autoestima, sexualidad e imagen corporal, el diagnóstico y los tratamientos para el cáncer de mama ${ }^{14}$. Las pacientes experimentan una profunda insatisfacción con la imagen corporal, que incluye la experiencia de sentirse mutilada, avergonzada, diferente, menos atractiva y femenina ${ }^{15-16}$. La imagen corporal se ve claramente afectada, tanto por la asimetría provocada por la cirugía, como por los efectos secundarios de los tratamientos, tales como alopecia, aumento/disminución del peso corporal o quemaduras en la zona irradiada. Los cambios en la apariencia física pueden deteriorar la relación de pareja y las relaciones sexuales, siendo frecuente la disminución de las mismas o la aparición de algún tipo de trastorno sexual ${ }^{17-20}$.

Por ello, la intervención psicológica en la imagen corporal en cáncer ha adquirido mucha importancia y representa un reflejo de la necesidad que tienen las pacientes de recibir una atención sanitaria que recoja el impacto emocional que provoca su diagnóstico y las dificultades de adaptación que surgen como resultado de los tratamientos ${ }^{21}$.

En sus relaciones personales surgen dificultades. Las mujeres asocian los tratamientos y sus consecuencias a tener cáncer, y de ahí surge un temor al estigma y se promueve el aislamiento social $^{22-23}$. Aunque en el discurso de nuestras pacientes se aprecie la intención de los demás de reducir el malestar de la paciente, ellas no lo viven así. Todo ello, lleva a las pacientes a evitar con frecuencia el contacto social y a aislarse, teniendo en cuenta además que no quieren preocupar a la familia y a los amigos con su malestar físico y psicológico.

En la familia surgen cambios en los roles, la mujer afectada pasa de ser la cuidadora principal de personas dependientes a cargo a ser cuidada por el resto de miembros familiares rompiendo con los estereotipos sociales y culturales asociados al rol de la mujer ${ }^{24}$.

En un estudio cualitativo sobre la vivencia de mujeres con cáncer de mama ${ }^{25}$ concluyen que el apoyo social otorgado por amigos, familiares o personas que han pasado por situaciones similares, fue fundamental en el proceso de afrontamiento de la enfermedad. Otras investigaciones han destacado la relevancia del 
apoyo social de la familia ${ }^{26-28}$, de la pareja $^{29} y$ del grupo de pares ${ }^{30}$ para hacer frente a secuelas psicológicas y sociales derivadas del cáncer, mejorando el nivel de estrés, el ajuste a la enfermedad y la calidad de vida de la paciente ${ }^{31}$.

A nivel sociocultural existe una línea de pensamiento representada, por ejemplo,7 en libros de autoayuda ${ }^{32}$ que plantea que las emociones positivas y el deseo de curarse tienen mucha influencia sobre el resultado de la enfermedad así como estados emocionales negativos podrían empeorar el proceso e incluso causar la muerte. Si bien existen ciertas investigaciones acerca de la influencia de factores de personalidad y emocionales en la aparición y evolución del cáncer ${ }^{33}$ no existe una evidencia clara a nivel científico ${ }^{34}$.

Dicho discurso social que se transmite a través de los medios de comunicación y el entorno inmediato produce en estas mujeres paradójicamente sentimientos de intenso malestar, ya que la mayoría se encuentran tristes, sienten apatía, ansiedad y tienen miedo y no pueden hacer nada para dejar de experimentar estos sentimientos. Todo ello, les induce a pensar que sus reacciones no son normales, que están llevando a cabo un mal afrontamiento e incluso que están poniendo en peligro el proceso de tratamiento y sus vidas. En algunas publicaciones divulgativas sobre casos de personas conocidas que han sufrido esta enfermedad, parece transmitirse el mensaje de que para curarse basta con querer y poner mucho empeño en ello y poco más, confundiendo y exigiendo al paciente en un momento en que ello puede servir para culpabilizarlo o deprimirlo más ${ }^{35}$. Holland y Lewis ${ }^{36}$ usan el término "tiranía del pensamiento positivo" para explicarlo y dicen: “... para muchos pacientes, el cáncer es la experiencia más difícil y aterradora a la que se han enfrentado. Todas esas supercherías, que proclaman que si uno no tiene una actitud positiva y está deprimido el tumor crece más rápidamente, invalidan las reacciones naturales y comprensibles de las personas ante algo que amenaza su vida”.

A nivel de los servicios sanitarios se sabe de la necesidad de información del paciente como uno de los déficits más comunes en la prestación de asistencia sanitaria, y entendiéndose que, para informar hay que saber comunicar ${ }^{37}$. La incertidumbre, el miedo y la ausencia de expresión de sentimientos hacen quelas pacientes y sus familiares estén ansiosos y sometidos a gran estrés. La mujer con cáncer de mama desea conocer y saber acerca de su enfermedad para poder controlar su vida, aclarar situaciones, resolver conflictos, terminar proyectos, dictar voluntades, disculparse e incluso despedirse en determinadas ocasiones; por lo que un buen manejo de la información por parte del personal sanitario, así como destreza y habilidad en comunicación, son fundamentales ${ }^{38}$.

En la experiencia con mujeres con cáncer de mama, el profesional debe tener habilidades técnicas y de comunicación relacional que son básicas y en las que se fundamenta la relación médico-paciente ${ }^{39}$.

A nivel de identidad se conoce que tanto la enfermedad como los tratamientos alteran el funcionamiento cotidiano y los roles establecidos además de la identidad personal, produciendo un duelo en múltiples aspectos. El cáncer puede situar a la persona en un espacio simbólico que le categoriza socialmente y le dota de características específicas hasta tal punto que, en ocasiones, la persona puede quedar socialmente resumida, reducida y anulada por la simple etiqueta diagnóstica ${ }^{40}$.

Para muchas mujeres son los senos lo primero que las define como mujeres y la pérdida de un 
pecho equivale a la pérdida de la feminidad ${ }^{41}$. Además, el pecho está relacionado fuertemente con la sexualidad y el atractivo físico además de con la maternidad y la lactancia ${ }^{42}$.

Frases como "esta no soy yo, quiero volver a ser la persona que era antes" son frecuentes en la consulta, frases que, hacen reflexionar sobre el papel que la identidad juega en los procesos de adaptación a la enfermedad. Se podría analizar cómo ha cambiado la visión que tienen de sí mismas las afectadas por cáncer como protagonistas de su mundo, abriendo la oportunidad de integrar en un mismo marco de experiencia dos eventos esenciales que puede generar la enfermedad: el sufrimiento y el crecimiento personal ${ }^{40}$. Es precisamente en el discurso de nuestras pacientes donde se descubre esta nueva reconstitución de la identidad que se va co-construyendo en la dinámica grupal a través de sus interacciones y procesos personales.

De la literatura que se refiere a cambios tras la enfermedad se ha de tener en cuenta que el diagnóstico de cáncer no genera en sí mismo un cambio en sentido positivo, sino los procesos cognitivos asociados a la percepción de amenaza a la propia vida ${ }^{43}$. Se podría decir que tras la enfermedad la persona confirma su fortaleza para superar dificultades por haber sobrevivido a lo peor, se siente más cercana y unida a los demás ante las dificultades, hay una voluntad para dejarse ayudar o utilizar el apoyo social que antes había ignorado. Muchas mujeres afirman que no consideran su vida más insatisfactoria que antes, sino que su vida actual es diferente. Esta forma de vida distinta tiene algunos aspectos que empeoran respecto a su calidad de vida previa, pero existen otros tantos aspectos que mejoran, como puede ser el cambio de valores y de sentido vital, que hacen que se perciba el mundo desde otro enfoque. En los períodos de confusión e incertidumbre que caracterizan al cáncer, las personas pueden buscar activamente un significado en un intento de entender porque les ha ocurrido. La terapia facilita la reflexión dela propia vida, pudiéndose convertir la enfermedad en un punto de inflexión, a partir de la cual hacer cambios en un sentido positivo ${ }^{4-46}$. Esto es acorde al discurso de nuestras participantes y se extrae de sus verbalizaciones algunos cambios positivos tras la enfermedad. De las áreas referidas por Tedeschi y Calhoun ${ }^{43}$ al menos cuatro coinciden en el discurso de las usuarias: mejora de las relaciones interpersonales, nuevas posibilidades, percepción de mayor fortaleza personal y apreciación por la vida.

En cuanto al análisis de la encuesta de satisfacción, las impresiones recogidas en la última sesión y en la consulta de valoración tras el desarrollo del grupo se pueden concluir varios aspectos.

Uno de los más importantes es que las pacientes consiguieron sentirse reflejada en el resto de participantes del grupo, lo que implica encontrar una validación a las propias emociones $y$ experiencias. Se trata de "saber que una no es la única", hay más personas que sufren esta enfermedad y sobre todo que se sienten de la misma manera. Todo ello parece llevar a una normalización del proceso, de forma que dejan de sentir que llevan a cabo un afrontamiento patológico. Así, las pacientes refieren encontrar en el grupo un nivel de comprensión que es difícil obtener fuera. El espacio grupal puede llegar a suponer una experiencia emocional correctiva ${ }^{47}$ en lo que a relaciones interpersonales se refiere. Así, una mujer reconoce que le ha permitido volver a confiar en la gente, dado que tras malas experiencias en su entorno tras el diagnóstico, la sinceridad de las compañeras le permitió abrirse y comprobar que no la juzgaban. Parece que esto le está permitiendo actualmente "reinventarse" y reconstruir su vida y su futuro. 
Sin embargo, las pacientes no solo recibieron del grupo validación y normalización, sino que las experiencias de las demás dieron lugar a una movilización de los recursos propios para enfrentarse a las situaciones. Una de ellas comenta que ver cómo las demás iban afrontando los problemas le hizo pensar que ella también podía hacerlo. En ocasiones, las soluciones aportadas en el grupo servían de guía para cada persona.

Además, relatan que el verse reflejadas en las demás dio lugar a un aumento de conciencia acerca de una misma y de sus estrategias, como por ejemplo una mujer que dice que la intervención le permitió "poder mirarse de forma más objetiva y ajustada". Y por último, se observa que en el grupo se generaban dinámicas de cuidado y apoyo cuando alguna de ellas se encontraba peor (planteándole aspectos positivos, sugiriendo soluciones a su problema, etc.).

En general, se puede decir que el grupo ha servido para cambiar la relación con la enfermedad. La mayoría de las pacientes habían tenido experiencias previas con el cáncer, ya fuese en familiares o amigos. Esto hace que a la carga de significado social que la palabra cáncer tiene, se unan multitud de asociaciones personales. El poder hablar de todo ello e ir modificando en grupo los contenidos relacionados con la enfermedad, permitió a varias de las pacientes reducir su miedo al cáncer y a la recidiva

Para finalizar, se exponen a continuación algunas de las implicaciones clínicas de los resultados encontrados.

Como ya apuntaban trabajos previos $^{9-12}$, tras un proceso de enfermedad y tratamiento en el cáncer de mama se producen cambios drásticos en relación a la propia imagen y la identidad: no sólo por cómo se ven las mujeres sino por cómo ellas sienten que las ven los demás. Acompañarlas con una actitud de respeto $y$ cuidado en el proceso de enfrentarse a su nueva realidad corporal, en la pérdida de sus anteriores roles vitales, en la construcción de nuevos roles, así como hacer partícipes de este proceso a las personas que conforman su entorno íntimo, son temas que deben formar parte del tratamiento integral de las personas con cáncer de mama. Se confirman las alteraciones de las dinámicas relacionales apuntadas en otros estudios ${ }^{48-50}$, por lo que se torna fundamental detectar las posibles dificultades que surgen por miedos a la reacción de una misma o del otro y proporcionar espacios de encuentro y apoyo que posibiliten que todo esto pueda hablarse, elaborarse y enfrentarse con las personas de su entorno significativo.

Resulta fundamental manejar con cautela el discurso basado en el optimismo y el ánimo infundado con personas que sostienen una carga importante de malestar real. El intento de animar a personas que experimentan la necesidad de ser escuchadas en su malestar puede generar el efecto contrario al deseado: desalentar, culpabilizar o hacer enfadar a aquella persona a la que se intenta dar ánimos. El hecho de posibilitar conversaciones sobre el malestar y enfrentarse a la realidad del mismo puede generar efectos deseables (por ejemplo, de manera indirecta una actitud más positiva).

Según los cambios observados en las mujeres que participaron en este grupo terapéutico, $y$ en congruencia con estudios previos ${ }^{51,8}$, es positivo proporcionar espacios de encuentro entre mujeres con experiencias vitales similares para facilitar el apoyo mutuo, la comprensión y la normalización del malestar. Esto facilita el avance y afrontamiento conjunto de las dificultades inherentes al proceso oncológico. 
Por lo general, las mujeres se sienten agradecidas y bien tratadas por los equipos sanitarios que están a su cargo. Sin embargo, les falta información fiable en relación a temas prácticos. La queja sobre la falta de cuidado de la privacidad y la intimidad de las pacientes durante las pruebas y el tratamiento, así como la descoordinación percibida entre los distintos profesionales de los equipos, repercuten negativamente en el bienestar de las pacientes. A pesar delos avances yel aumento de concienciación sobre la importancia del tratamiento integral del cáncer, la experiencia de estas mujeres advierte que aún queda trabajo por hacer. Sería interesante la elaboración de un breve protocolo informativo que abordase temas relevantes a considerar en los tratamientos oncológicos (la exposición al sol, la depilación, el uso de ropa interior, la sexualidad, etc.) que pudiera ser realizado por personal sanitario que estuviese en contacto con ellas antes de recibir dichos tratamientos. Por último, son necesarias reuniones entre profesionales de diferentes especialidades en las que estas necesidades puedan ser abordadas y se facilite así una verdadera atención conjunta a todas las esferas que puedan afectar a la calidad de vida de estas pacientes.

\section{AGRADECIMIENTOS}

A Javier Llamazares y María Eugenia Díez por haber compartido esta experiencia de Atención Continuada. A Sonia González, por la revisión del artículo que ha aportado ideas enriquecedoras y por haber sido una compañera y $R$ mayor estupenda.

\section{DECLARACIÓN SOBRE CONFLICTOS DE INTERESES}

Las autoras declaran no tener ningún conflicto de interés en la elaboración y publicación del presente trabajo.

\section{REFERENCIAS BIBLIOGRÁFICAS}

1. Asociación Española Contra el Cáncer (AECC). Incidencia del cáncer de mama, actualizada en 2014. [Internet]. España. Disponible en: https://www. aecc.es/sobreelcancer/cancerporlocalizacion/ cancermama/paginas/incidencia.aspx.

2. Cruzado JA, Olivares ME. Tratamiento psicológico de los pacientes con cáncer. Madrid: Pirámide; 2000.

3. Remor E, Arranz P, Ulla S. El psicólogo en el ámbito hospitalario. Bilbao: Desclee de Brouwer; 2003.

4. Given C, Given B, Rahbar M, Jeon S, McCorkle R, Cimprich B, et al. Effect of a cognitive behavioural intervention on reducing symptom severity during chemotherapy. J Clin Oncol. 2004; 22 (3): 507-16.

5. Lengacher CA, Johnson-Mallard V, PostWhite J, Moscoso, MS, Jacobsen PB, Klein TW, et al. Randomized controlled trial of mindfulness-based stress reduction (MBSR) for survivors of breast cancer. Psychooncology. 2009; 18 (12): 1261-72.

6. Rehse B, Pukrop R. Effects of psychosocial interventions on quality of life in adult cancer patients: meta analysis of 37 published controlled outcome studies. Patient Educ Couns. 2003; 50 (2):179-86.

7. Smith SK, Hemdon JE, Lyerly HK, Coan A, Wheeler JL, Staley T, et al. Correlates of quality of life-related outcomes in breast cancer patients participating in the Pathfinders pilot study. Psychooncology. 2011; 20 (5): 559-64.

8. Rodríguez E, Font A. Eficacia de la terapia de grupo en cáncer de mama: Evolución de las emociones desadaptativas. Psicooncología 2013; $10(2-3): 275-87$.

9. Ochoa C, Sumalla E, Maté J, Castejón V, Rodríguez A, Blanco I, et al. Psicoterapia positiva grupal en cáncer. Hacia una atención psicosocial integral del superviviente de cáncer. Psicooncología. 2010; 7(1): 7-34. 
10. Medín G. Supervivientes de cáncer infantil. Efectos en el tiempo. Un estudio cualitativo. Psicooncología. 2009; 6(2-3): 429-43.

11. Navarro E, Limonero J, Maté J, Gómez-Romero MJ. Necesidades de comunicación e información en el paciente oncológico superviviente. Psicooncología. 2010; 7(1): 127-41.

12. Hill-Schnipper H. Life after breast cancer. J Clin Oncol. 2001; 19(15): 3581-4.

13. Berterö C, Chamberlain M. Breast Cancer Diagnosis and Its Treatment Affecting the Self: A Meta-Synthesis. Cancer Nurs. 2007; 30(3): 194-202.

14. Narváez A, Rubiños C, Cortés-Funes F, Gómez $\mathrm{R}$, García A. Valoración de la eficacia de una terapia grupal cognitivo-conductual en la imagen corporal, autoestima, sexualidad y malestar emocional (ansiedad y depresión) en pacientes con cáncer de mama. Psicooncología. 2008; 5(1): 93-102.

15. Ganz PA, Rowland JH, Desmond K. Life after breast cancer: understanding women's healthrelated quality of life and sexual functioning in patients with breast cancer. J Clin Oncol. 1998; 16 (2):501-14.

16. Fobair P, Stewart SL, Chang S, D'Onofrio C, Banks PJ, Bloom JR. Body image and sexual problems in young women with breast cancer. Psychooncology. 2006; 15 (7):579-94.

17. Die-Trill M, Die-Trill A. El cáncer de mama. En: Die Trill M, editor. Psicooncología. Madrid: ADES, 2003; p. 165-84.

18. Al-Ghazal SK, Fallowfield L, Blomey RW. Comparison of psychological aspects and patient satisfaction following breast conserving surgery, simple mastectomy and breast reconstruction. Eur J Cancer. 2000; 36(15):1938-43.

19. Arora NK, Gustafson DH, Hawkins RP, McTavish F, Cella DF, Pingree S, et. al. Impact of surgery and chemotherapy on the quality of life of younger women with breast carcinoma: a prospective study. Cancer. 2001; 92(5):1288-98.

20. Rowland JH, Desmond KA, Meyerowitz BE, Belin TR, Wyatt GE, Gant PA. Role of breast reconstructive surgery in physical and emotional outcomes among breast cancer survivors. J Natl Cancer Inst. 2000; 92(17):1422-9.

21. Sebastián J, Bueno MJ, Mateos N. Apoyo emocional y calidad de vida en mujeres con cáncer de mama. Factores psicosociales en cáncer de mama. Madrid: Instituto de la Mujer. Ministerio de Trabajo y Asuntos Sociales.2002.

22. Frith H, Harcourt D, Fussell A. Anticipating an altered appearance: women undergoing chemotherapy treatment for breast cancer. Eur J Oncol Nurs. 2007; 11 (5):385-91.

23. Harcourt D, Frith H. Women's experiences of an altered appearance during chemotherapy: an indication of cancer status. J Health Psychol. 2008; 13 (5):597- 606.

24. Sumalla EC, Castejón V, Ochoa C, Blanco I. ¿Por qué las mujeres con cáncer de mama deben estar guapas y los hombres con cáncer de próstata pueden ir sin afeitar? Oncología, disidencia y cultura hegemónica. Psicooncología. 2013; 10(1): 7-56.

25. Barra C, Hidalgo M, Muñoz C, Reyes P, Ubilla P. Vivencia del diagnóstico y tratamiento en mujeres con cáncer de mama y su relación con el autoconcepto. [Tesis para optar al grado de Licenciado en Psicología]. Chile: Universidad San Sebastián. Facultad de Ciencias Sociales; 2008. 
26. Arora N, Finney L, Gustafson D, Moser R, Hawkins R. Perceived helpfulness and impact of social support provided by family, friends, and health care providers to women newly diagnosed with breast cancer. Psychooncology. 2007; 16 (5): 474-486.

27. Carrasco P, Cea L. Construcción de significados asociados a los patrones de interacción familiar en mujeres con cáncer de mama, y su relación con el afrontamiento de la enfermedad. [Trabajo para optar al grado de Magíster en Psicología Clínica]. Santiago de Chile: Universidad Adolfo Ibáñez; 2007.

28. Luszczynska A, Mohamed N, Schwarzer R. Selfefficacy and social support predict benefit finding 12 months after cancer surgery: The mediating role of coping strategies. Psycho, Health \& Med. 2005; 10 (4): 365-375.

29. Tamblay A, Bañados B. Psicooncología del cáncer de mama. [Memoria para optar al Título de Psicólogo]. Chile: Universidad de Chile. Departamento de Psicología, Facultad de Ciencias Sociales; 2004.

30. Durá E, Hernández S. Los grupos de auto-ayuda como apoyo social en el cáncer de mama. Las asociaciones de mujeres mastectomizadas de España. Bol de Psic. 2003; 78: 21-39.

31. García F, Rincón P. Prevención de Sintomatología Postraumática en Mujeres con Cáncer de Mama: Un Modelo de Intervención Narrativo. Ter psic. 2011; 29 (2): 175-183.

32. Byrne R. El secreto. Barcelona: Urano; 2007.

33. Eysenck HJ. Cancer, personality and stress: prediction and prevention. Adv Behav Res Ther. 1994; 16(3): 167-215

34. Petticrew M, Bell R, Hunter D. Influence of psychological coping on survival and recurrence in people with cancer: systematic review. BMJ. 2002; 325 (7372): 1066-69.

35. Alonso C, Bastos A. Intervención Psicológica en Personas con Cáncer. Cli Cont. 2011; 2 (2): 187-207.
36. Holland J, Lewis S. La cara humana del cáncer. Vivir con esperanza, afrontar la incertidumbre. Barcelona: Herder; 2003.

37. Kirk P, Kirk I, Kristjanson LJ. What do patients receiving palliative care for cancer and their families want to be told? A canadian and australian qualitative study. BMJ. 2004; 328 (7452): 1343.

38. Mallinger JB, Griggs JJ, Shields CG. Patientcentered care and breast cancer survivors' satisfaction with information. Patient Educ Couns. 2005; 57(3): 342-349.

39. Gámez GG. The nurse-patient relationship as a caring relationship. Nurs Sci Q. 2009; 22(2): 126-127.

40. SumallaE, OchoaC, GilF,Blanco I.Sobretránsitos, identidades y crecimiento postraumático en cáncer hereditario. Psicooncología. 2009; 6(1): 7-25.

41. Carver CS, Pozo-Kaderman C, Price A, Noriega V, Harris S, Derhagopian RP, et al. Concerns about aspects of body image and adjustment to early stage breast cancer. Psychosom Med. 1998; 60 (2): 168-174.

42. Sebastián J, Manos D, Bueno MJ, Mateos N. Imagen corporal y autoestima en mujeres con cáncer de mama participantes en un programa de intervención psicosocial. Clín y Sal. 2007; 18 (2): 137-161.

43. Calhoum LG, Cann A, Tedeschi G, McMillan J. A Correlational test of the relationship between posttraumatic growth, religion, and cognitive processing. J Trauma Stress. 2000. 13 (3): 521-7.

44. Bower JE, Meyerowitz B, Desmond KA, Bernaards C, Rowland JH, Ganz PA. Perceptions of positive meaning and vulnerability following breast cancer: Predictors and outcomes among long-term breast cancer survivors. Ann Behav Med. 2005; 29 (3): 236- 45. 
45. Schoen EG, Nicholas DR. Existential meaning through illness: the case of patients with breast cancer. J Psychosoc Oncol. 2004; 22: 61-76.

46. Tártaro J, Roberts J, Nosarti C, Crayford T, Luecken L, David A. Who benefits? Distress, adjustment and benefit-finding among breast cancer survivors. J Psychosoc Oncol. 2005; 23 (2-3): 45-64.

47. Alexander F, French TM. Psychoanalytic Therapy: Principles and Application. New York: Ronald; 1946.

48. Holmberg S, Scott L, Alexy W, Fife B. Relationship Issues of Women With Breast Cancer. Cancer Nurs. 2001; 24 (1): 53-60.
49. Marcus Lewis F, Hammond MA, Woods NF. The family's functioning with newly diagnosed breast cancer in the mother: The development of an explanatory model. J Behav Med.1993; 16(4): 351-70.

50. Takahashi M, Kai I. Sexuality after breast cancer treatment: Changes and coping strategies among Japanese survivors. Soc Sci Med. 2005; 61 (6):1278-90

51. Goodwin PJ, Leszcz M, Ennis M, Koopmans J, Vincent L, Guther H, et al. The Effect of Group Psychosocial Support on Survival in Metastatic Breast Cancer. N Engl J Med. 2001; 345 (24):1719-26. 